\title{
Discovery and validation of PZP as a novel serum biomarker for screening lung adenocarcinoma in type 2 diabetes mellitus patients
}

Jiayue Yang ${ }^{1 \dagger}$, Cheng Yang ${ }^{2 \dagger}$, Hong Shen ${ }^{1}$, Wenjun Wu ${ }^{1}$, Zhen Tian ${ }^{3}$, Qinghua Xu' ${ }^{1}$ Cuiping Cao ${ }^{1}$, Shugao Ye ${ }^{4}$, Le Ban ${ }^{4}$, Xin Tong ${ }^{3}$ and Jie Mei ${ }^{F^{*}}$

\begin{abstract}
Background: Patients with type 2 diabetes mellitus (T2DM) have an increased risk of suffering from various malignancies. This study aimed to identify specific biomarkers that can detect lung adenocarcinoma (LAC) in T2DM patients for the early diagnosis of LAC.

Methods: The clinical information of hospitalized T2DM patients diagnosed with various cancers was collected by reviewing medical records in Wuxi People's Hospital Affiliated to Nanjing Medical University from January 1, 2015, to June 30, 2020. To discover diagnostic biomarkers for early-stage LAC in the T2DM population, 20 samples obtained from 5 healthy controls, 5 T2DM patients, 5 LAC patients and 5 T2DM patients with LAC (T2DM + LAC) were subjected to sequential windowed acquisition of all theoretical fragment ion mass spectrum (SWATH-MS) analysis to identify specific differentially-expressed proteins (DEPs) for LAC in patients with T2DM. Then, these results were validated by parallel reaction monitoring MS (PRM-MS) and ELISA analyses.

Results: Lung cancer was the most common malignant tumor in patients with T2DM, and LAC accounted for the majority of cases. Using SWATH-MS analysis, we found 13 proteins to be unique in T2DM patients with early LAC. Two serum proteins were further validated by PRM-MS analysis, namely, pregnancy-zone protein (PZP) and insulin-like growth factor binding protein 3 (IGFBP3). Furthermore, the diagnostic values of these proteins were validated by ELISA, and PZP was validated as a novel serum biomarker for screening LAC in T2DM patients.

Conclusions: Our findings indicated that PZP could be used as a novel serum biomarker for the identification of LAC in T2DM patients, which will enhance auxiliary diagnosis and assist in the selection of surgical treatment at an early stage.
\end{abstract}

Keywords: Biomarker, Lung adenocarcinoma, Type 2 diabetes mellitus, Mass spectrum

\footnotetext{
*Correspondence: meijie1996@njmu.edu.cn

†jiayue Yang and Cheng Yang contributed equally to this article.

${ }^{5}$ Department of Oncology, Wuxi People's Hospital Affiliated to Nanjing Medical University, No. 299 Qingyang Road, Wuxi 214023, China

Full list of author information is available at the end of the article
}

\section{Background}

Diabetes mellitus is a group of metabolic disorders characterized by chronic hyperglycemia caused by complicated etiologies. Statistical data organized by the International Diabetes Federation revealed that there were approximately 387 million people worldwide who had diabetes mellitus in 2014, which is estimated to

(c) The Author(s) 2021. This article is licensed under a Creative Commons Attribution 4.0 International License, which permits use, sharing, adaptation, distribution and reproduction in any medium or format, as long as you give appropriate credit to the original author(s) and the source, provide a link to the Creative Commons licence, and indicate if changes were made. The images or other third party material in this article are included in the article's Creative Commons licence, unless indicated otherwise in a credit line to the material. If material is not included in the article's Creative Commons licence and your intended use is not permitted by statutory regulation or exceeds the permitted use, you will need to obtain permission directly from the copyright holder. To view a copy of this licence, visit http://creativeco mmons.org/licenses/by/4.0/. The Creative Commons Public Domain Dedication waiver (http://creativecommons.org/publicdomain/ zero/1.0/) applies to the data made available in this article, unless otherwise stated in a credit line to the data. 
increase to 592 million in 2035 [1]. Diabetes mellitus occurs when the body cannot produce enough insulin or use insulin effectively. The former is defined as type 1 diabetes mellitus (T1DM), and the latter is type 2 diabetes mellitus (T2DM) [2]. Increasing evidence has revealed that T2DM is associated not only with microvascular complications (including nephropathy, retinopathy and neuropathy) and macrovascular complications (such as cardiovascular diseases) [3] but also with the oncogenesis and development of multiple types of cancer, including lung cancer, breast cancer and pancreatic cancer $[4,5]$.

Cancer is gradually becoming the first cause of mortality worldwide with growing numbers of estimated new cases and deaths each year [6]. Increasing evidence supports a direct association between T2DM and cancer with higher risks of cancer morbidity and mortality, especially for some of the most common malignancies [7]. To date, several mechanisms underlying the cancer-T2DM association have been explored, uncovering dysregulations of the insulin-like growth factor (IGF) system as the most important paradigm $[7,8]$. However, despite the higher risk of cancer morbidity in the T2DM population, reliable biomarkers for screening and early diagnosis of specific types of cancer in T2DM patients have not yet been discovered.

Mass spectrum (MS)-dependent strategies offer novel insights for the identification and validation of diseaserelated biomarkers $[9,10]$. For example, Geyer et al. developed a plasma proteome analysis pipeline using label-free quantitative MS, which detected $284 \pm 5$ proteins containing $>40 \mathrm{FDA}$-approved biomarkers without removing high-abundance proteins [11]. Sequential windowed acquisition of all theoretical fragment ion mass spectrum (SWATH-MS) is a newly developed strategy using a data-independent acquisition (DIA) method with high quantitative accuracy and reproducibility [12]. Using this strategy, increasing numbers of disease biomarkers have been identified, and novel criteria for disease typing based on proteomics have been established [13-15].

In this research, we first collected clinical information of hospitalized T2DM patients diagnosed with cancer and found that lung cancer was the most common malignant tumor in patients with T2DM in our cohort, with lung adenocarcinoma (LAC) accounting for the majority of cases. Using SWATH-MS and parallel reaction monitoring MS (PRM-MS) analyses, we discovered and preliminarily validated pregnancy zone protein (PZP) and insulin-like growth factor binding protein 3 (IGFBP3) as potential biomarkers. ELISA analysis was next used to further validate these biomarkers, and PZP was determined as a novel serum biomarker for screening LAC in
T2DM patients, which will enhance auxiliary diagnosis and assist in the selection of early surgical therapeutics for LAC.

\section{Methods \\ Patients and sample description}

The clinical information of hospitalized T2DM patients diagnosed with cancer was collected by reviewing medical records in Wuxi People's Hospital Affiliated to Nanjing Medical University from January 1, 2015, to June 30,2020 . The following two cohorts were used to discover and validate biomarkers (Fig. 1a): In the discovery set, a total of 20 serum samples from 5 healthy controls, 5 T2DM patients, 5 LAC patients at TNM stage 1 and 5 T2DM patients with LAC at TNM stage 1 (T2DM+LAC), which were submitted to SWATHMS analysis; besides, 20 serum samples from T2DM patients and 20 serum samples from T2DM patients with LAC at TNM stage 1 were submitted for PRM-MS and ELISA analysis. In the validation set, 20 serum samples from T2DM patients and 20 serum samples from T2DM patients with LAC at TNM stage 1 were collected for ELISA analysis. Before analysis, the serum samples were kept at $-80{ }^{\circ} \mathrm{C}$ until use. The study was approved by the Ethical Committee at Wuxi People's Hospital Affiliated to Nanjing Medical University, and the study was performed according to the Declaration of Helsinki.

\section{SWATH-MS analysis \\ Sample preparation}

An Agilent Multiple Affinity Removal LC Column (Human 14) (Agilent, CA, USA) was used to remove high-abundance proteins in accordance with the protocol to obtain a low-abundance component solution in the serum sample. A $5 \mathrm{kD}$ ultrafiltration tube was used for ultrafiltration and concentration, and one-fold volume of SDT lysis was added into the system, which was incubated in a water bath at $100{ }^{\circ} \mathrm{C}$ for $10 \mathrm{~min}$ and centrifuged at $14,000 \times \mathrm{g}$ for $15 \mathrm{~min}$. The supernatant was extracted for protein quantification using a $\mathrm{BCA}$ kit, and the samples were subpackaged and stored at $-80^{\circ} \mathrm{C}$.

\section{FASP digestion}

DTT was added to $200 \mu \mathrm{g}$ of protein solution collected from each sample to reach a final concentration of $100 \mathrm{mM}$, and the samples were incubated in a water bath at $100{ }^{\circ} \mathrm{C}$ for $5 \mathrm{~min}$. UA buffer $(200 \mu \mathrm{L})$ was then added, and the samples were mixed and transferred to a $30 \mathrm{kD}$ ultrafiltration centrifuge tube. The samples were centrifuged at $12,500 \times \mathrm{g}$ for $25 \mathrm{~min}$, and the filtrate was discarded (this step was repeated twice). IAA buffer $(100 \mu \mathrm{L} ; 100 \mathrm{mM}$ IAA in UA) was then added, 
a

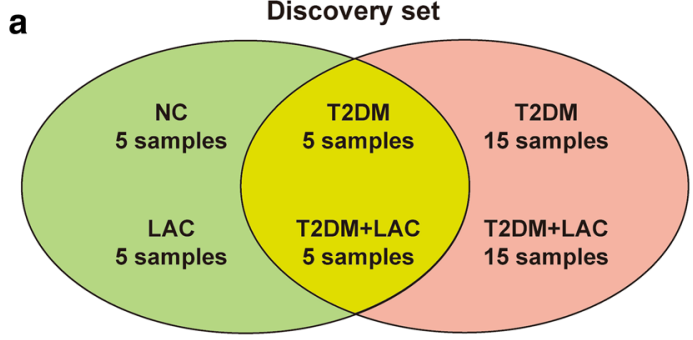

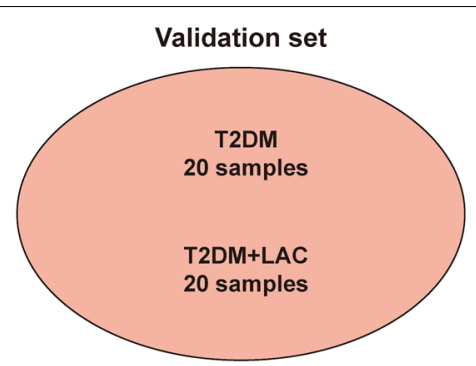

b

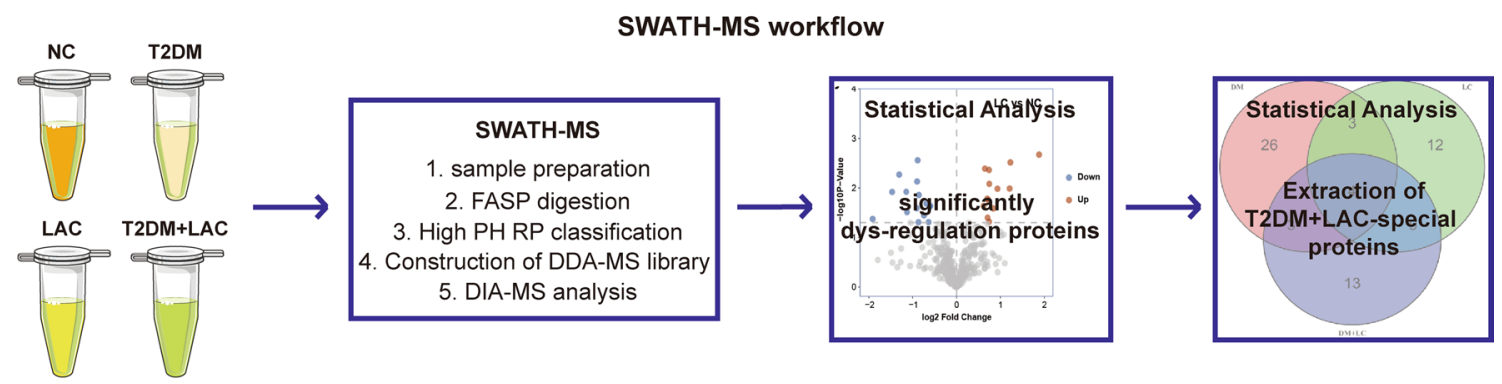

PRM-MS and ELISA workflow
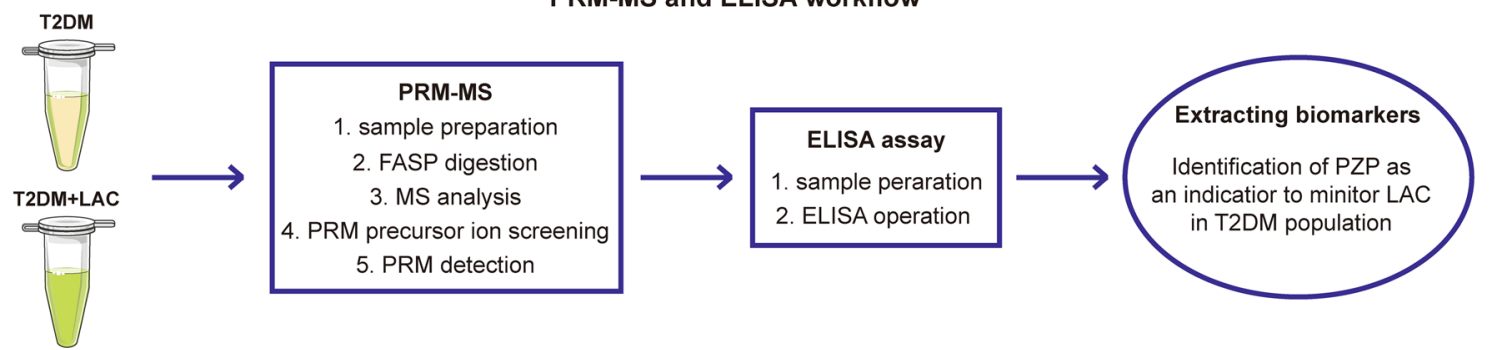

Fig. 1 Overview of the study design. a Schematic diagram of serum specimens included in this study. $\mathbf{b}$ Schematic representation of the steps followed for the screening of the diagnostic proteins for LAC in T2DM patients (top) and the validated procedures (bottom)

and the samples were shaken at $600 \mathrm{rpm}$ for $1 \mathrm{~min}$. The samples were allowed to react at room temperature for $30 \mathrm{~min}$ in the dark and then centrifuged at $12,500 \times \mathrm{g}$ for $25 \mathrm{~min}$. UA buffer $(100 \mu \mathrm{L})$ was then added, and the samples were centrifuged at $12,500 \times \mathrm{g}$ for $15 \mathrm{~min}$ (this step was repeated twice). Then, $40 \mathrm{mM} \mathrm{NH}_{4} \mathrm{HCO}_{3}$ $(100 \mu \mathrm{L})$ was added, and the samples were centrifuged at $12,500 \times \mathrm{g}$ for $15 \mathrm{~min}$ (this step was repeated twice). Trypsin buffer ( $40 \mu \mathrm{L} ; 4 \mu \mathrm{g}$ of trypsin in $40 \mu \mathrm{L}$ of $40 \mathrm{mM} \mathrm{NH}_{4} \mathrm{HCO}_{3}$ ) was then added, and the samples were shaken at $600 \mathrm{rpm}$ for $1 \mathrm{~min}$ and placed at $37^{\circ} \mathrm{C}$ for 16-18 h. The collection tube was replaced, and the samples were centrifuged at $12,500 \times \mathrm{g}$ for $15 \mathrm{~min}$ followed by the addition of $20 \mu \mathrm{L}$ of $40 \mathrm{mM} \mathrm{NH}_{4} \mathrm{HCO}_{3}$ and centrifugation at $12,500 \times \mathrm{g}$ for $15 \mathrm{~min}$ to collect the filtrate. A C18 cartridge was used to desalt the peptides. After the peptides were dried, they were reconstituted with $40 \mu \mathrm{L}$ of $0.1 \%$ formic acid solution.

\section{High PH RP classification}

The peptide mixtures of all samples were submitted for fractionation using the Agilent 1260 infinity II HPLC system. Buffer A solution consisted of $10 \mathrm{mM}$ $\mathrm{HCOONH}_{4}$ and $5 \% \mathrm{ACN}(\mathrm{pH} 10)$, and solution B consisted of $10 \mathrm{mM} \mathrm{HCOONH}{ }_{4}$ and $85 \% \mathrm{ACN}(\mathrm{pH} 10)$. The chromatographic column was balanced with buffer $\mathrm{A}$, and the sample was loaded by the autosampler onto the chromatographic column (XBridge Peptide $\mathrm{BEH}$ C18 Column, $130 \AA, 5 \mu \mathrm{m}, 4.6 \mathrm{~mm} \times 100 \mathrm{~mm}$; Waters, MA, USA) for separation with a flow rate of $1 \mathrm{~mL} / \mathrm{min}$. 
The liquid phase gradient was as follows: linear gradient of $5 \%$ B to $45 \%$ B within 40 min with a column temperature maintained at $30^{\circ} \mathrm{C}$. In total, 36 components were collected, and each component was dried in a vacuum concentrator for use. The sample was lyophilized, reconstituted with $0.1 \%$ formic acid aqueous solution and combined into 12 fractions.

\section{Construction of DDA-MS library}

From each fraction, $6 \mu \mathrm{L}$ was removed and added to $2 \mu \mathrm{L}$ of $10 \times$ iRT standard peptide, and $2 \mu \mathrm{L}$ of each sample was separated with nano-LC and analyzed by online electrospray tandem MS. The complete liquid-mass tandem system consisted of a liquid system (Waters Acquity UPLC; Waters, MA, USA) and an MS system (Q-Exactive HF; Thermo Fisher Scientific, MA, USA). Buffer A consisted of $0.1 \%$ formic acid aqueous solution, and buffer B consisted of $0.1 \%$ formic acid acetonitrile aqueous solution (acetonitrile was $80 \%$ ). The sample was separated by an analytical column (Thermo Fisher Scientific, MA, USA; Acclaim PepMap C18, $75 \mu \mathrm{m} \times 25 \mathrm{~cm}$ ) at a flow rate of $200 \mathrm{~nL} / \mathrm{min}$ with the following gradient: $0-5 \mathrm{~min}, 1 \%$ B; 5-95 min, $1 \%$ B to $28 \%$ B; $95-110 \mathrm{~min}, 28 \%$ B to $38 \%$ B; $110-115 \mathrm{~min}, 38 \%$ B to $100 \% \mathrm{~B}$; and $115-120 \mathrm{~min}, 100 \%$ B. The electrospray voltage was $2.0 \mathrm{kV}$. The MS parameters were set as follows: (1) MS: scan range $(\mathrm{m} / \mathrm{z})=350$ 1600 , resolution $=60,000$, AGC target $=3 \mathrm{e} 6$, maximum injection time $=50 \mathrm{~ms}$ and filter dynamic exclusion: exclusion duration $=30 \mathrm{~s}$; and (2) dd-MS2: isolation window $=4 \mathrm{~m} / \mathrm{z}$, resolution $=15,000$, AGC target $=5 \mathrm{e}$, maximum injection time $=80 \mathrm{~ms}$ and $\mathrm{NCE}=30 \%$. The MS raw data were analyzed and searched by Spectronaut Pulsar X (version 12, Biognosys AG), and a spectral database was established. The standard for library construction was $1 \%$ precursor FDR and 1\% peptide FDR.

\section{DIA-MS analysis}

From each fraction, $6 \mu \mathrm{L}$ was removed and added to 2 $\mu \mathrm{L}$ of $10 \times \mathrm{iRT}$ standard peptide, and $2 \mu \mathrm{L}$ of each sample was separated with nano-LC and analyzed by online electrospray tandem MS. The entire experimental system was an Orbitrap Q Exactive HF mass spectrometer (Thermo Fisher Scientific, MA, USA) connected in series with a Waters Acquity UPLC (Waters, MA, USA) system. Buffer A consisted of $0.1 \%$ formic acid aqueous solution, and buffer B consisted of $0.1 \%$ formic acid acetonitrile aqueous solution (acetonitrile was 80\%). The sample was separated by an analytical column (Thermo Fisher Scientific, MA, USA; Acclaim PepMap C18, $75 \mu \mathrm{m} \times 25 \mathrm{~cm}$ ) at a flow rate of $200 \mathrm{~nL} / \mathrm{min}$ using the following nonlinear increasing gradient: $0-5 \mathrm{~min}, 1 \%$ B; $5-95$ min, $1 \%$ B to $28 \%$ B; $95-110$ min, $28 \%$ B to $38 \%$
B; $110-115 \mathrm{~min}, 38 \% \mathrm{~B}$ to $100 \% \mathrm{~B}$; and $115-120 \mathrm{~min}$, $100 \% \mathrm{~B}$. The electrospray voltage was $2.0 \mathrm{kV}$. The MS parameters were set as follows: (1) MS: scan range $(\mathrm{m} / \mathrm{z})=350-1250$, resolution $=120,000, \quad$ AGC target $=3 \mathrm{e} 6$ and maximum injection time $=20 \mathrm{~ms}$; and (2) DIA: resolution $=30,000$, AGC target $=1 \mathrm{e} 6$, maximum injection time $=$ auto and $\mathrm{NCE}=25.5,27,30$. The original MS data and the default parameters of Spectronaut Pulsar X were used to analyze the DIA data. The protein qualitative standard was a precursor threshold of $1.0 \%$ FDR. Serum proteins compared between the two specified groups with a threshold of fold change $(\mathrm{FC}) \geq 1.50$ or $\leq 0.67$ and $P$ value $\leq 0.05$ were considered as differentially-expressed proteins (DEPs).

\section{PRM-MS analysis}

\section{Sample preparation and FASP digestion}

The expression of DEPs was preliminarily verified by PRM, which was a target proteomic strategy. For PRM assays, the methods for sample preparation and FASP digestion were the same as previously described for SWATH-MS analysis.

\section{MS analysis}

The same mass of peptides from each sample was extracted and mixed well, and $2 \mu \mathrm{g}$ of each sample was separated with nano-LC and analyzed by online electrospray tandem MS. The complete liquid-mass tandem system was composed of a liquid system (Easy nLC system; Thermo Fisher Scientific, MA, USA) and an MS system (Q-Exactive; Thermo Fisher Scientific, MA, USA). Buffer A was composed of $0.1 \%$ formic acid aqueous solution, and buffer B was composed of $0.1 \%$ formic acid acetonitrile aqueous solution (acetonitrile was $80 \%$ ). The sample was separated by an analytical column (Thermo Fisher Scientific, MA, USA; Acclaim PepMap RSLC $50 \mu \mathrm{m} \times$ $15 \mathrm{~cm}$, nano viper, $\mathrm{P} / \mathrm{N} 164943)$ at a flow rate of $300 \mathrm{~nL} /$ min using the following nonlinear increasing gradient: $0-1 \mathrm{~min}, 2 \% \mathrm{~B}$ to $8 \% \mathrm{~B} ; 1-46 \mathrm{~min}, 8 \% \mathrm{~B}$ to $28 \% \mathrm{~B}$; $46-56 \mathrm{~min}, 28 \%$ B to $40 \% \mathrm{~B} ; 56-57 \mathrm{~min}, 40 \%$ B to $90 \%$ B; and $57-60 \mathrm{~min}, 90 \% \mathrm{~B}$.

The samples were chromatographed and analyzed by a $Q$ Exactive mass spectrometer with the following parameters; analysis time of $60 \mathrm{~min}$; detection method was positive ion; precursor ion scan range of $350-1500 \mathrm{~m} / \mathrm{z}$, resolution of the primary MS was 60,000; AGC target was 3e6; and primary maximum IT was $45 \mathrm{~ms}$. The massto-charge ratios of peptides and peptide fragments were collected according to the following method: 10 fragment patterns (MS2 scan) were collected after each full scan (MS2 scan); MS2 activation type was HCD; isolation window was $2 \mathrm{~m} / \mathrm{z}$; MSMS resolution rate was 15,000 , 
AGC target was 2e5; secondary Maximum IT was $45 \mathrm{~ms}$; and normalized collision energy was $27 \mathrm{eV}$.

\section{PRM precursor ion screening}

Proteome Discoverer 2.1 (Thermo Fisher Scientific, MA, USA) software was used to convert the original map files (.raw files) generated by $\mathrm{Q}$ Exactive into.mgf files, which were submitted to the MASCOT2.6 server for database retrieval through the built-in tools of the software. The database used was Uniprot_HomoSapiens_20386_20180905. The reliable protein screening criterion was peptide FDR $\leq 0.01$.

\section{PRM detection}

Each sample $(2 \mu \mathrm{g})$ was separated by nano-LC and analyzed by online electrospray tandem MS. The complete liquid-mass tandem system was composed of a liquid system (Easy nLC system; Thermo Fisher Scientific, MA, USA) and an MS system (Q-Exactive; Thermo Fisher Scientific, MA, USA). Buffer A was composed of $0.1 \%$ formic acid aqueous solution, and buffer $B$ was composed of $0.1 \%$ formic acid acetonitrile aqueous solution (acetonitrile was $80 \%$ ). The sample was separated by an analytical column (Thermo Fisher Scientific, MA, USA; Acclaim PepMap RSLC $50 \mu \mathrm{m} \times 15 \mathrm{~cm}$, nano viper, P/N164943) at a flow rate of $300 \mathrm{~nL} / \mathrm{min}$ using the following nonlinear increasing gradient: $0-1 \mathrm{~min}, 2 \% \mathrm{~B}$ to $8 \% \mathrm{~B} ; 1-46 \mathrm{~min}, 8 \%$ B to $28 \% \mathrm{~B} ; 46-56 \mathrm{~min}, 28 \%$ B to $40 \% \mathrm{~B}$; $56-57 \mathrm{~min}, 40 \%$ $\mathrm{B}$ to $90 \% \mathrm{~B}$; and $57-60 \mathrm{~min}, 90 \% \mathrm{~B}$.

The MS parameters were set as follows: (1) Full-MS: scan range $(\mathrm{m} / \mathrm{z})=350-1500$, resolution $=60,000$, AGC target $=1 \mathrm{e} 6$ and maximum injection time $=50 \mathrm{~ms}$; and (2) PRM: resolution $=15,000$, AGC target $=1 \mathrm{e} 5$, maximum injection time $=50 \mathrm{~ms}$, loop count $=14$; isolation window $=1.6 \mathrm{~m} / \mathrm{z}$ and $\mathrm{NCE}=27 \%$. Skyline software was used for analysis of PRM data.

\section{ELISA analysis}

The concentrations of PZP (Catalog No. DY8280-05; R\&D Systems, MN, USA) and IGFBP3 (Catalog No. DGB300; R\&D Systems, MN, USA) in serum were quantified with commercially available ELISA kits according to the manufacturer's protocol. Most samples were assayed in duplicates, and the average values were reported as $\mathrm{pg} / \mathrm{mL}$ or $\mathrm{ng} / \mathrm{mL}$. The linear correlation between the PRM-MS and ELISA results was calculated using Pearson's correlation analysis.

\section{Analysis of public data}

The data of PZP mRNA expression in the TCGA database was obtained from the Xena website. The correlations between PZP expression and immune cell infiltration were determined by the TIMER database [16]. Besides, the summary of PZP protein was consulted in the HPA database $[17,18]$.

\section{Statistical analysis}

Statistical analysis was mainly performed in SPSS (v26.0) and GraphPad Prism (v.8.0). Most of the data between the two groups were presented as means \pm SDs (Std. Deviations) if not noted and were compared by Student's t-test or the Mann-Whitney test. Correlation analysis was evaluated by Pearson's correlation analysis. Receiveroperating characteristic (ROC) analysis was used to assess the specificity and sensitivity of the biomarkers, and the area under the ROC curve (AUC) was estimated for each individual protein. For all analyses, $P$ values less than 0.05 were considered statistically significant.

\section{Results}

\section{Distribution of tumor location and subtype of lung cancer in T2DM patients}

Previous research has indicated that lung cancer is the most common concomitant malignant tumor among patients with diabetes [1]. Thus, to further confirm the distribution of tumor location, we collected clinical information of hospitalized T2DM patients diagnosed with cancers from January 1, 2015, to June 30, 2020. After analyzing the distribution, we found that lung cancer was the highest proportion of malignant tumors (20.84\%) followed by digestive tract cancers (colorectum: $12.81 \%$, stomach: $12.32 \%$, and liver: $6.18 \%$ ) (Table 1 ). We next analyzed the histological types of T2DM patients with lung cancer. The proportion of histological types was as follows: adenocarcinoma (60.62\%), squamous carcinoma (13.86\%), small cell carcinoma (3.69\%), mixed carcinoma (1.47\%), neuroendocrine carcinoma $(0.88 \%)$, magnocellular carcinoma $(0.29 \%)$ and other histological types (0.88\%) (Table 2). Overall, LAC accounted for the most common tumor in T2DM patients and should be monitored and diagnosed early.

\section{Patient characteristics and study design}

Before we screened the potential biomarker that could differentiate LAC in T2DM patients, we first tried to compare the general pathological parameters in the main two groups in the whole set consisting of 40 serum samples from T2DM patients and 40 serum samples from T2DM patients with LAC. In the T2DM group, there were 23 males and 17 females with an average age of $61.05 \pm 9.78$ years and an average fasting plasma glucose (FPG) of $7.95 \pm 1.91 \mathrm{mmol} / \mathrm{L}$. In the T2DM + LAC group, there were 19 males and 21 females with an average age of $64.68 \pm 7.10$ years and an average FPG of 
Table 1 Distribution of tumor location and gender in patients with T2DM and cancer

\begin{tabular}{|c|c|c|c|c|c|c|c|}
\hline \multirow[t]{2}{*}{ Location } & \multirow[t]{2}{*}{ Age } & \multicolumn{4}{|c|}{ Gender } & \multirow[t]{2}{*}{ Total } & \multirow[t]{2}{*}{ Proportion } \\
\hline & & Male & & Female & & & \\
\hline Lung & $66.66 \pm 8.36$ & 448 & $66.08 \%$ & 230 & $33.92 \%$ & 678 & $20.84 \%$ \\
\hline Colorectum & $69.46 \pm 10.01$ & 250 & $59.95 \%$ & 167 & $40.05 \%$ & 417 & $12.81 \%$ \\
\hline Stomach & $68.75 \pm 8.83$ & 288 & $71.82 \%$ & 113 & $28.18 \%$ & 401 & $12.32 \%$ \\
\hline Liver & $67.80 \pm 11.26$ & 152 & $75.62 \%$ & 49 & $24.38 \%$ & 201 & $6.18 \%$ \\
\hline Prostate & $73.79 \pm 7.97$ & 193 & $100.00 \%$ & 0 & $0.00 \%$ & 193 & $5.93 \%$ \\
\hline Breast & $65.97 \pm 11.45$ & 4 & $2.27 \%$ & 172 & $97.73 \%$ & 176 & $5.41 \%$ \\
\hline Blood & $64.80 \pm 11.30$ & 97 & $58.08 \%$ & 70 & $41.92 \%$ & 167 & $5.13 \%$ \\
\hline Pancreas & $68.91 \pm 9.24$ & 97 & $63.82 \%$ & 55 & $36.18 \%$ & 152 & $4.67 \%$ \\
\hline Bladder & $70.02 \pm 9.13$ & 103 & $84.43 \%$ & 19 & $15.57 \%$ & 122 & $3.75 \%$ \\
\hline Kidney & $65.72 \pm 10.50$ & 73 & $66.97 \%$ & 36 & $33.03 \%$ & 109 & $3.35 \%$ \\
\hline Esophagus & $69.38 \pm 8.44$ & 67 & $67.00 \%$ & 33 & $33.00 \%$ & 100 & $3.07 \%$ \\
\hline Lymphoma & $67.11 \pm 9.64$ & 53 & $56.38 \%$ & 41 & $43.62 \%$ & 94 & $2.89 \%$ \\
\hline Thyroid & $53.52 \pm 10.75$ & 35 & $43.21 \%$ & 46 & $56.79 \%$ & 81 & $2.49 \%$ \\
\hline Gallbladder \& biliary & $70.13 \pm 10.07$ & 40 & $53.33 \%$ & 35 & $46.67 \%$ & 75 & $2.30 \%$ \\
\hline Uterus & $63.57 \pm 12.02$ & 0 & $0.00 \%$ & 74 & $100.00 \%$ & 74 & $2.27 \%$ \\
\hline Multiple sites & $71.39 \pm 10.33$ & 32 & $69.57 \%$ & 14 & $30.43 \%$ & 46 & $1.41 \%$ \\
\hline Head and neck & $67.00 \pm 10.45$ & 24 & $77.42 \%$ & 7 & $22.58 \%$ & 31 & $0.95 \%$ \\
\hline Ovary & $63.48 \pm 8.22$ & 0 & $0.00 \%$ & 25 & $100.00 \%$ & 25 & $0.77 \%$ \\
\hline Small intestine & $70.86 \pm 8.48$ & 12 & $57.14 \%$ & 9 & $42.86 \%$ & 21 & $0.65 \%$ \\
\hline Skin & $70.88 \pm 10.52$ & 10 & $62.50 \%$ & 6 & $37.50 \%$ & 16 & $0.49 \%$ \\
\hline Brain & $65.13 \pm 10.87$ & 8 & $53.33 \%$ & 7 & $46.67 \%$ & 15 & $0.46 \%$ \\
\hline Ureter & $74.82 \pm 6.66$ & 7 & $63.64 \%$ & 4 & $36.36 \%$ & 11 & $0.34 \%$ \\
\hline Thymus & $58.30 \pm 9.09$ & 4 & $40.00 \%$ & 6 & $60.00 \%$ & 10 & $0.31 \%$ \\
\hline Other & $66.64 \pm 10.15$ & 24 & $61.54 \%$ & 15 & $38.46 \%$ & 39 & $1.20 \%$ \\
\hline Total & $67.70 \pm 10.13$ & 2021 & $62.11 \%$ & 1233 & $37.89 \%$ & 3254 & $100.00 \%$ \\
\hline
\end{tabular}

Table 2 Distribution of histological classification and gender in patients with T2DM and lung cancer

\begin{tabular}{|c|c|c|c|c|c|c|c|}
\hline \multirow[t]{2}{*}{ Histological classifications } & \multirow[t]{2}{*}{ Age } & \multicolumn{4}{|c|}{ Gender } & \multirow[t]{2}{*}{ Total } & \multirow[t]{2}{*}{ Proportion } \\
\hline & & Male & & Fema & & & \\
\hline Adenocarcinoma & $66.74 \pm 8.17$ & 256 & $62.29 \%$ & 155 & $37.71 \%$ & 411 & $60.62 \%$ \\
\hline Squamous carcinoma & $65.89 \pm 8.27$ & 61 & $64.89 \%$ & 33 & $35.11 \%$ & 94 & $13.86 \%$ \\
\hline Small cell carcinoma & $65.80 \pm 7.05$ & 15 & $60.00 \%$ & 10 & $40.00 \%$ & 25 & $3.69 \%$ \\
\hline Mixed carcinoma & $66.20 \pm 6.75$ & 8 & $80.00 \%$ & 2 & $20.00 \%$ & 10 & $1.47 \%$ \\
\hline Neuroendocrine carcinoma & $67.17 \pm 7.49$ & 4 & $66.67 \%$ & 2 & $33.33 \%$ & 6 & $0.88 \%$ \\
\hline Magnocellular carcinoma & $69.00 \pm 5.66$ & 2 & $100.00 \%$ & 0 & $0.00 \%$ & 2 & $0.29 \%$ \\
\hline Other & $68.67 \pm 7.15$ & 6 & $100.00 \%$ & 0 & $0.00 \%$ & 6 & $0.88 \%$ \\
\hline Unknown & $67.05 \pm 9.57$ & 96 & $77.42 \%$ & 28 & $22.58 \%$ & 124 & $18.29 \%$ \\
\hline Total & $66.66 \pm 8.36$ & 448 & $66.08 \%$ & 230 & $33.92 \%$ & 678 & $100.00 \%$ \\
\hline
\end{tabular}

$7.41 \pm 2.55 \mathrm{mmol} / \mathrm{L}$. There were no statistically significant differences in sex, age and FPG between the two groups $(\mathrm{P}>0.05)$ (Table 3). Besides, there was also no significant differences in therapeutic regimens for hypoglycemia between these two groups $(\mathrm{P}>0.05)$ (Table 3). Moreover, we compared the concentrations of the most commonly used tumor biomarkers in the clinic between these two groups. The results showed that there were no significant 
Table 3 Comparison of general information between two groups

\begin{tabular}{llll}
\hline General information & T2DM & T2DM + LAC & P value \\
\hline Gender (male/female) & $23 / 17$ & $19 / 21$ & 0.371 \\
Hypoglycemic therapy (A/B) $^{\mathrm{a}}$ & $27 / 13$ & $26 / 14$ & 0.813 \\
Age (years) & $61.05 \pm 9.78$ & $64.68 \pm 7.10$ & 0.137 \\
AFP $(\mathrm{ng} / \mathrm{ml})$ & $2.70 \pm 1.17$ & $3.21 \pm 1.57$ & 0.101 \\
CEA $(\mathrm{ng} / \mathrm{ml})$ & $2.11 \pm 1.09$ & $3.27 \pm 3.72$ & 0.304 \\
CA125 (U/ml) & $9.50 \pm 4.86$ & $12.34 \pm 15.66$ & 0.693 \\
CA199 (U/ml) & $14.41 \pm 10.44$ & $27.51 \pm 68.25$ & 0.994 \\
FPG (mmol/L) & $7.95 \pm 1.91$ & $7.41 \pm 2.55$ & 0.287 \\
\hline
\end{tabular}

${ }^{a} A$, insulin or insulin-dependent therapies; $B$, insulin-independent therapies or no treatment

differences in serum AFP $(P=0.101)$, CEA $(P=0.304)$, CA125 $(\mathrm{P}=0.693)$ and CA199 $(\mathrm{P}=0.994)$ levels between the T2DM+LAC group and the T2DM group (Table 3 ). These results suggested that the identification of novel biomarkers is urgently needed for the detection of LAC in T2DM patients.

Considering the limited values of common tumor biomarkers in T2DM patients, we next performed SWATHMS, PRM-MS and ELISA analyses to identify and validate novel biomarkers for the detection of LAC in T2DM patients. The overall strategy and simplified workflow are shown in Fig. 1b. Briefly, 20 samples obtained from 5 healthy controls, 5 T2DM patients, 5 LAC patients and 5 T2DM patients with LAC were submitted for SWATHMS analysis to identify DEPs specific for LAC in patients with T2DM. These results were next validated by PRMMS and ELISA analysis. Moreover, the validation set consisting of 20 serum samples from T2DM patients and 20 serum samples from T2DM patients with LAC were collected for ELISA analysis and further validation.

\section{Identification of differentially expressed proteins by SWATH-MS analysis}

Using SWATH-MS analysis, we analyzed global protein changes in serum samples from 20 patients $(5$ healthy controls, 5 T2DM patients, 5 LAC patients and 5 T2DM + LAC patients). A total of 70 proteins were identified as differentially expressed between these disease groups and the control group (Fig. $2 \mathrm{a}-\mathrm{c}$ ). As shown in Fig. 2d, the three protein lists from the above analysis (T2DM vs. normal, LAC vs. normal and T2DM + LAC vs. normal) were further compared to identify a small group of proteins that were differentially expressed only in the T2DM+LAC group. Overall, 13 proteins were found to be unique in patients with $\mathrm{T} 2 \mathrm{DM}+\mathrm{LAC}$
(Fig. 2d). Among these proteins, 7 candidates exhibited differential expression between the T2DM+LAC and T2DM groups, including 2 upregulated proteins and 5 downregulated proteins (Tables 4 and 5). To arrange the samples according to similarities in protein expression patterns, we performed a hierarchical cluster analysis of the 70 DEPs as previously described [19]. Cluster analysis indicated a clear separation of the four groups (Fig. 2e).

\section{Verification of selected candidate proteins by PRM-MS ELISA analyses}

Of the 13 proteins identified as DEPs in patients with T2DM+LAC by SWATH-MS analysis, 7 proteins showed significant dysregulation between T2DM + LAC and T2DM, including CCD87, FHR1, FRPD2, HBB, IGFBP3, PZP, and ZN350 (Table 5). We next used targeted PRM-MS to provide high sensitivity relative peptide quantification for validation. A total of 4 proteins were detected by PRM-MS, and significant differential expression of 2 of these candidate proteins was confirmed, namely, PZP and IGFBP3 (Fig. 3a-d, Additional file 1: Figure S1).

We next validated the protein abundance changes of PZP and IGFBP3 using commercially available antibodies and ELISA kits. The concentration-dependent standard curve is shown in Additional file 2: Figure S2. To evaluate the feasibility of developing an assay that could be more easily deployed in a clinical environment, we assessed the transferability of the PRM-MS-based results to ELISA. The levels of PZP and IGFBP3 were quantified by commercially available ELISA kits, and the correlation with the results obtained by PRM-MS was evaluated. The results showed a linear correlation for PZP but not IGFBP3 (Fig. 4a, Additional file 3: Figure S3). In addition, the level of PZP between the T2DM + LAC and T2DM groups was significantly different in the discovery set, the validation set and the whole set, and the ROC analysis indicated an AUC of 0.742 (Fig. 4b-e). However, no significant difference was observed in IGFBP3 levels between these two groups (Additional file 3: Figure S3). In summary, detection of PZP level provides enough sensitivity and specificity, and it merits further validation in larger cohort samples.

\section{Discussion}

As two common chronic non-communicable diseases, more and more studies have realized the correlation between lung cancer and T2DM. In a meta-analysis, Lee et al. systematically analyzed 34 observational studies and found that after adjusting for smoking and other variables, T2DM was an independent risk factor for the 

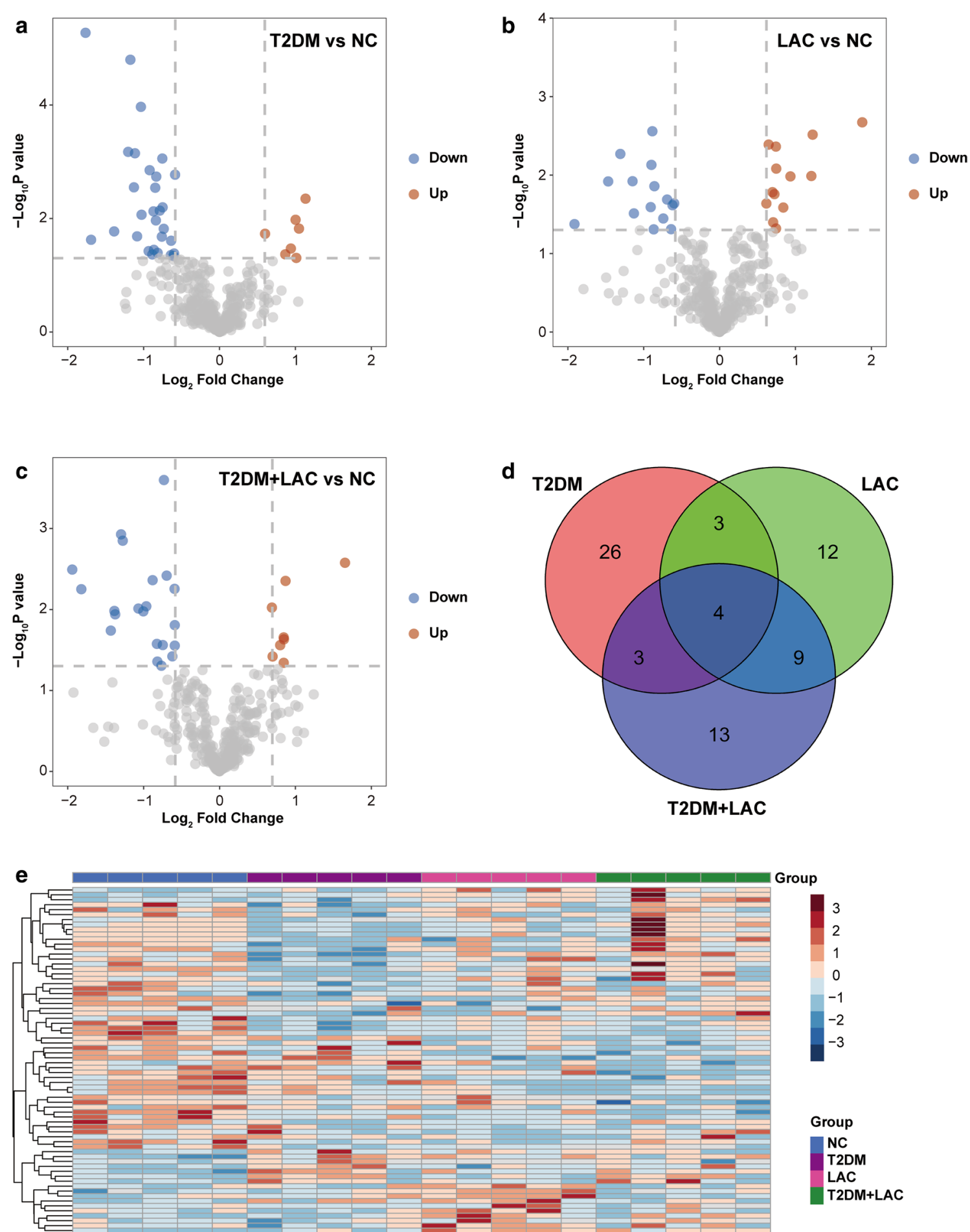

Fig. 2 Identification of unique serum proteins in T2DM patients with LAC. a-c Volcano plot showing the DEPs in the T2DM, LAC and T2DM + LAC patients compared to healthy controls. $\mathbf{d}$ Venn diagram of the 70 differentially expressed proteins in the serum samples from the four comparisons (T2DM vs. NC, LAC vs. NC and T2DM + LAC vs. NC). In total, 13 proteins were specifically expressed in the T2DM + LAC group. e Hierarchical cluster analysis of the 70 DEPs in the NC, T2DM, LAC, and T2DM + LAC serum samples. Note: There were five samples in each group. Pseudocolors indicate differential expression (red, upregulation; blue, downregulation) 
Table 4 List of serum differential proteins identified by SWATH-MS in patients with T2DM + LAC and T2DM

\begin{tabular}{lll}
\hline Protein & Accession & Protein description \\
\hline ADIPO & Q15848 & Adiponectin \\
CCD87 & Q9NVE4 & Coiled-coil domain-containing protein 87 \\
CXCL7 & P02775 & Platelet basic protein \\
FBLN1 & P23142 & Fibulin-1 \\
FHR1 & Q03591 & Complement factor H-related protein 1 \\
FRPD2 & Q68DX3 & FERM and PDZ domain-containing protein 2 \\
HBB & P68871 & Hemoglobin subunit beta \\
HV145 & A0A0A0MS14 & Immunoglobulin heavy variable 1-45 \\
IGFBP3 & P17936 & Insulin-like growth factor-binding protein 3 \\
LV316 & A0A075B6K0 & Immunoglobulin lambda variable 3-16 \\
PZP & P20742 & Pregnancy zone protein \\
SRGN & P10124 & Serglycin \\
ZN350 & Q9GZX5 & Zinc finger protein 350 \\
\hline
\end{tabular}

Table 5 Unpaired T-test of the quantitative proteomic results for protein expression in the T2DM + LAC group in comparison with the control and T2DM groups

\begin{tabular}{|c|c|c|c|c|}
\hline \multirow[t]{2}{*}{ Protein } & \multicolumn{2}{|c|}{ T2DM + LAC vs. control } & \multicolumn{2}{|c|}{$T 2 D M+L A C$ vs. T2DM } \\
\hline & Fold change & Pvalue & Fold change & $P$ value \\
\hline ADIPO & -1.873 & 0.004 & -1.629 & 0.199 \\
\hline CCD87 & -1.664 & $<0.001$ & -1.440 & $<0.001$ \\
\hline CXCL7 & -2.700 & 0.018 & -2.245 & 0.115 \\
\hline FBLN1 & -1.506 & 0.016 & -1.372 & 0.098 \\
\hline FHR1 & 1.613 & 0.010 & 1.510 & 0.025 \\
\hline FRPD2 & -2.097 & 0.010 & -1.751 & 0.035 \\
\hline $\mathrm{HBB}$ & -3.534 & 0.006 & -2.188 & 0.019 \\
\hline HV145 & -1.702 & 0.049 & -1.392 & 0.138 \\
\hline IGFBP3 & -1.765 & 0.044 & -2.371 & 0.003 \\
\hline PZP & 1.801 & 0.023 & 2.488 & 0.022 \\
\hline SRGN & 1.624 & 0.038 & 1.573 & 0.131 \\
\hline ZN350 & -1.679 & 0.028 & -1.810 & 0.006 \\
\hline
\end{tabular}

LV316 was excluded because of be missing in most samples

occurrence of lung cancer with a relative risk of 1.11 and a $95 \% \mathrm{CI}$ of 1.02 to 1.20 [20]. At the same time, T2DM is also related to the risk of lung cancer death. Tseng et al. conducted a prospective study of 244,920 T2DM patients with a 12-year follow-up and found that the LC mortality rate of T2DM patients was significantly higher [21]. In the present research, we systematically analyzed the distribution of tumor location and subtype of lung cancer in T2DM patients. The results revealed that lung cancer was the most common malignant tumor in patients with T2DM, with LAC accounting for the majority of cases. Moreover, unlike pancreatic cancer, which has the highest increased risk in patients with T2DM, the early diagnosis and treatment of lung cancer can significantly improve prognosis [22, 23]. Therefore, more strategies for the early screening of LAC in T2DM patients should be further explored.

Although cytology is the gold standard for the diagnosis of malignancies, serum biomarkers are also invaluable in the screening and auxiliary diagnosis of malignant tumors as well as monitoring curative effects [24, 25]. The serum proteome holds significant interest as a potential source of biomarkers and is an easily accessible fluid for auxiliary diagnosis. Four tumor biomarkers, including AFP, CEA, CA125 and CA199, are widely used in clinical practice. An observational study presented by Chen et al. revealed the association between the levels of these biomarkers and the tumor stage of LAC. Serum AFP was not correlated with $\mathrm{T}$ stage, $\mathrm{N}$ stage or $\mathrm{M}$ stage, but serum CEA and serum CA125 were positively correlated with $\mathrm{T}$ stage, $\mathrm{N}$ stage and M stage. Serum CA199 was not correlated with $\mathrm{T}$ stage but was positively correlated with $\mathrm{N}$ stage and $\mathrm{M}$ stage [26]. However, it is unknown whether these four biomarkers help to identify LAC in patients with T2DM. In our study, the results indicated that there were no significant differences in serum CEA, AFP, CA125 and CA199 levels between the T2DM + LAC group and the T2DM group, indicating an urgent need for the identification of promising biomarkers for the detection of LAC in T2DM patients.

The MS-dependent identification of serum biomarkers has recently emerged $[27,28]$. SWATH-MS is a newly developed technology, which combines the advantages and characteristics of traditional "shotgun" proteomics and selective reaction monitoring/multiple reaction monitoring (SRM/MRM) [12]. SWATH-MS technology can obtain all fragment information of all ions in the sample without omission and difference, while PRM technology can achieve the absolute quantification of protein expression. The combination of the two strategies can be used for the efficient, comprehensive and accurate screening of potential biomarkers [29, 30]. In this study, we performed SWATH-MS analysis to identify DEPs specific for LAC in patients with T2DM, and these potential biomarkers were validated by PRM-MS and ELISA analysis in the discovery and validation cohort.

To identify a small group of proteins that were differentially expressed in the T2DM + LAC group, we compared the three protein lists (T2DM+LAC vs. normal, T2DM vs. normal and LAC vs. normal) and identified 13 proteins that were unique in patients with T2DM $+\mathrm{LAC}$. Among these proteins, 7 candidates exhibited differential expression between the T2DM + LAC and T2DM groups. 

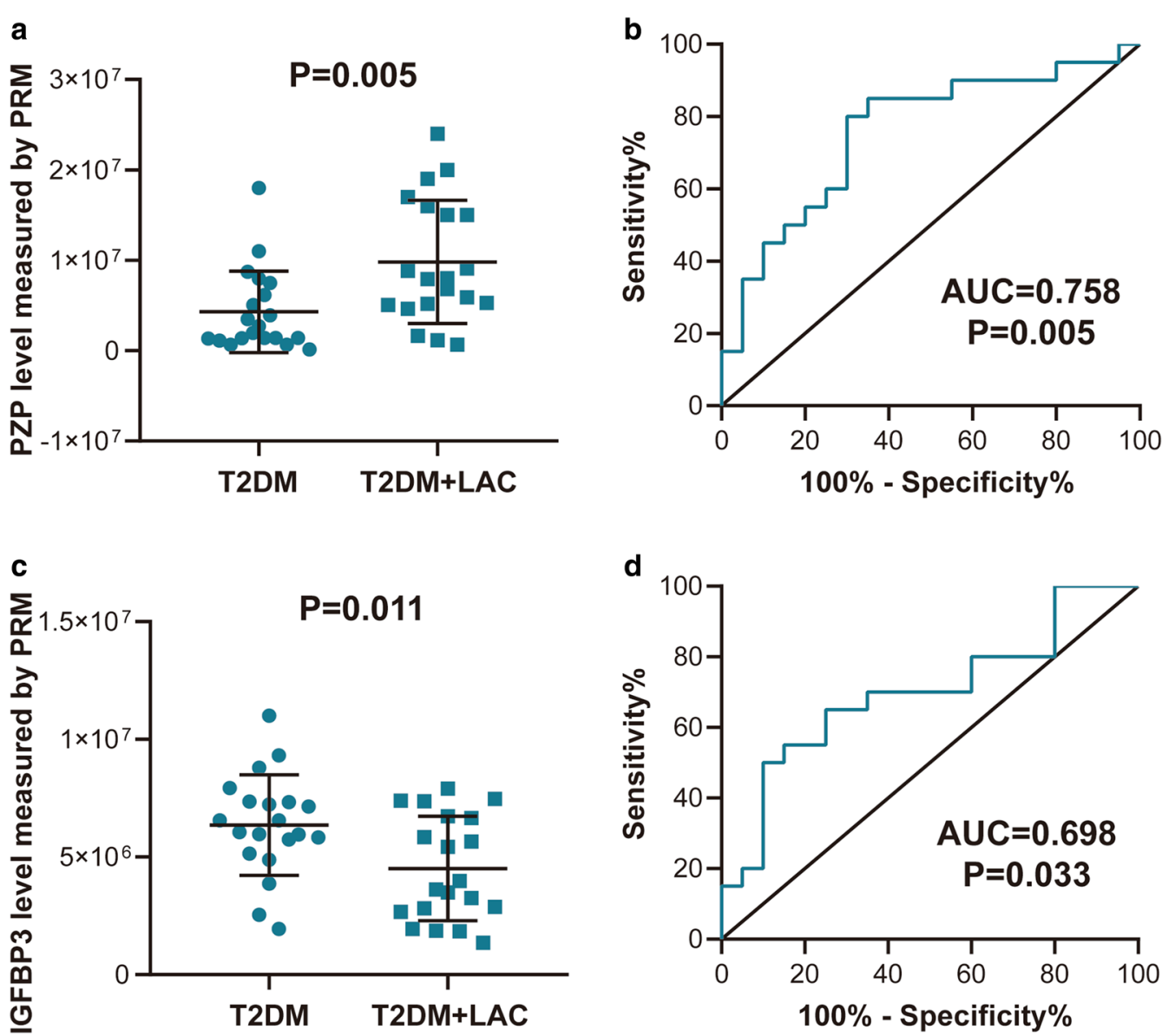

Fig. 3 Validation of selected candidate proteins by PRM-MS analysis. Differential expression of (a) PZP and (c) IGFBP3 in the T2DM + LAC and T2DM groups. ROC analysis of the diagnostic value of (b) PZP and (d) IGFBP3 for the detection of LAC in T2DM patients

To identify useful diagnostic indicators from these 7 proteins, we conducted further validation by PRM-MS. The results showed that 4 proteins were detected by PRM-MS and that significant differential expression of 2 of these candidate proteins was confirmed, namely, PZP and IGFBP3. As a first step toward clinical implementation, the diagnostic biomarker was assessed by ELISA. Immunoassays continue to be the preferred method for clinical validation and further application in clinical practice [31]. The PZP levels were significantly different between the T2DM + LAC and T2DM groups, and the ROC analysis indicated an AUC of 0.742 in the whole set. However, no significant difference in IGFBP3 levels was observed between these two groups.

PZP is associated with pregnancy, and it is produced in the liver, placenta and other tissues. The blood concentration of PZP increases during pregnancy [32].
Mechanically, elevated estrogen levels during pregnancy may regulate PZP levels [33]. Moreover, elevated PZP has been identified as an indicator associated with $P$. aeruginosa infection. Sputum but not serum concentrations of PZP have been significantly associated with the Bronchiectasis Severity Index, the frequency of exacerbations and symptoms [34]. Previous research has also uncovered the role of PZP in cancers. In hepatocellular carcinoma, PZP has low expression in tumor tissues, and the downregulation of PZP is correlated with poor clinical outcomes [35]. Our research identified and validated PZP as a novel serum biomarker for screening LAC in patients with T2DM by SWATH-MS, PRM-MS and ELISA analyses. Besides, we also analyzed the expression of PZP and its correlations with immune cell infiltration in lung cancer. The results showed that PZP mRNA was downregulated in lung cancer tissues and significantly correlated 

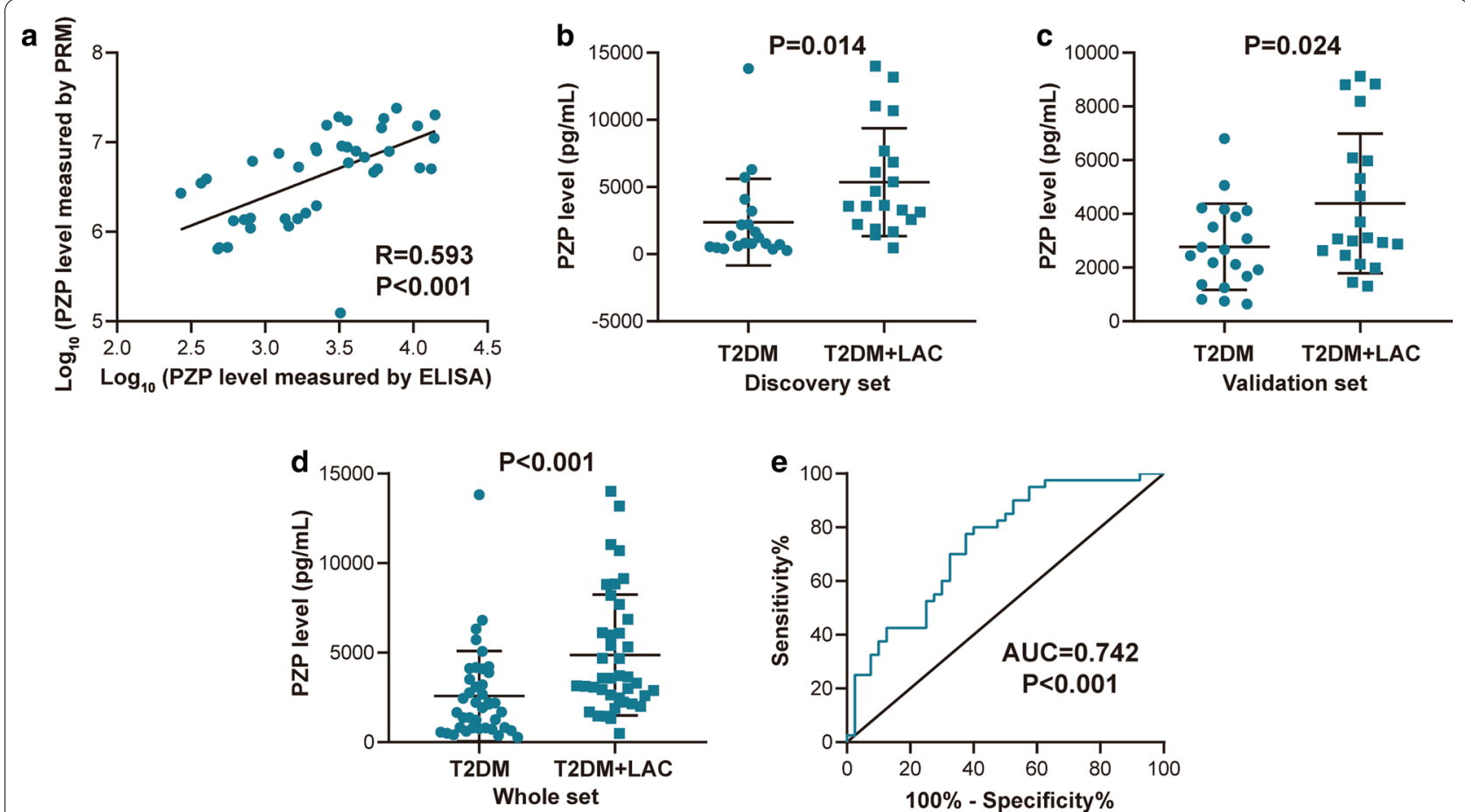

Fig. 4 Validation of selected candidate proteins by ELISA analysis. a Correlation between PRM-MS and ELISA assay results for PZP. $\mathbf{b}$ Differential expression of PZP in the T2DM + LAC and T2DM groups in the discovery set. $\mathbf{c}$ Differential expression of PZP in the T2DM+ LAC and T2DM groups in the validation set. $\mathbf{d}$ Differential expression of PZP in the T2DM + LAC and T2DM groups in the whole set. e ROC analysis of the diagnostic value of PZP for the detection of LAC in T2DM patients in the whole set

with immune cell infiltration (Additional file 4: Figure S4A-B). However, in the TCGA database, not all patients have T2DM before the diagnosis of lung cancer. Besides, the TCGA database only provides gene expression data at the mRNA level. Serum biomarkers are not only derived from tumor cell, but may also be released by tumorrelated immune cells [36]. According to the HPA database, PZP is highly expressed in immune cells, including $T$ cells and macrophages. In previous research, $P$. aeruginosa infection-induced PZP elevation was derived from neutrophils [34]. Therefore, serum PZP may be derived from tumor-related immune cells, but further studies still need to confirm the source of PZP and its diagnostic value by large-scale analysis.

\section{Conclusion}

In conclusion, the present results revealed that PZP could be used as a novel serum biomarker for the detection of LAC in T2DM patients, which will enhance auxiliary diagnosis at an early stage. However, the present study was conducted using a small sample size at a single center. Hence, the performance of the biomarker panel needs to be validated in a prospective, multicentric study with a higher number of patients.

\section{Abbreviations}

T1DM:Type 1 diabetes mellitus; T2DM: Type 2 diabetes mellitus; IGF: Insulinlike growth factor; MS: Mass spectrum; SWATH-MS: Sequential windowed acquisition of all theoretical fragment ion mass spectrum; DIA: Dataindependent acquisition; PRM-MS: Parallel reaction monitoring MS; LAC: Lung adenocarcinoma; IGFBP3: Insulin-like growth factor binding protein 3; T2DM + LAC:T2DM patients with LAC at TNM stage 1; FC: Fold change; DEPs: Differentially expressed proteins; ROC: Receiver-operating characteristic; AUC: The area under the ROC curve; FPG: Fasting plasma glucose; SRM/MRM: Selective reaction monitoring/multiple reaction monitoring.

\section{Supplementary Information}

The online version contains supplementary material available at https://doi. org/10.1186/s12935-021-01861-8.

Additional file 1: Figure S1. Validation of selected candidate proteins by PRM-MS analysis. Differential expression of (A) HBB and (B) CFHR1 in the T2DM+LAC and T2DM groups.

Additional file 2: Figure S2. Standard curve of ELISA assay for (A) PZP and (B) IGFBP3.

Additional file 3: Figure S3. Validation of IGFBP3 by ELISA analysis. (A) Correlation between PRM-MS and ELISA assay results for IGFBP3. (B) Differential expression of IGFBP3 in the T2DM+LAC and T2DM groups. 
Additional file 4: Figure S4. (A) The expression of PZP mRNA in lung cancer and (B) its correlations with immune cells infiltration.

\section{Acknowledgements}

None.

\section{Authors' contributions}

JM and JY proposed the experimental design. JY, CY, HS, ZT, QX, WW, and CC executed the experiments. JY, CY, SY, LB, and XT helped with data interpretation and analysis. JM, JY, and CY composed the manuscript. The final manuscript was reviewed and approved by all authors before submission. All authors read and approved the final manuscript.

\section{Funding}

This work was fouded by the General Project of Wuxi Health Commission (M202016), the Major Project of Wuxi Health Commission (Z202017) and the Taihu Talent Program (BJ2020011).

\section{Availability of data and materials}

The data used to support the findings of this study are available from the corresponding author upon request.

\section{Declarations}

\section{Ethical approval and consent to participate}

This study was approved by the Ethical Committee at Wuxi People's Hospital Affiliated to Nanjing Medical University.

\section{Consent for publication}

Each author approved the manuscript before submission for publication.

\section{Conflict of interest}

The authors declare no conflict of interest.

\section{Author details}

1 Department of Endocrinology, Wuxi People's Hospital Affiliated to Nanjing Medical University, Wuxi 214023, China. ${ }^{2}$ Department of Gastroenterology, Wuxi People's Hospital Affiliated to Nanjing Medical University, Wuxi 214023, China. ${ }^{3}$ Department of Clinical Laboratory, Wuxi People's Hospital Affiliated to Nanjing Medical University, Wuxi 214023, China. ${ }^{4}$ Department of Chest Surgery, Wuxi People's Hospital Affiliated to Nanjing Medical University, Wuxi 214023, China. ${ }^{5}$ Department of Oncology, Wuxi People's Hospital Affiliated to Nanjing Medical University, No. 299 Qingyang Road, Wuxi 214023, China.

Received: 24 January 2021 Accepted: 1 March 2021

Published online: 10 March 2021

\section{References}

1. Wang M, Hu RY, Wu HB, Pan J, Gong WW, Guo LH, et al. Cancer risk among patients with type 2 diabetes mellitus: a population-based prospective study in China. Sci Rep. 2015;5:11503.

2. Alberti KG, Zimmet PZ. Definition, diagnosis and classification of diabetes mellitus and its complications. Part 1: diagnosis and classification of diabetes mellitus provisional report of a WHO consultation. Diabet Med. 1998;15(7):539-53.

3. DeFronzo RA, Ferrannini E, Groop L, Henry RR, Herman WH, Holst JJ, et al. Type 2 diabetes mellitus. Nat Rev Dis Primers. 2015;1:15019.

4. Shlomai G, Neel B, LeRoith D, Gallagher EJ. Type 2 diabetes mellitus and cancer: the role of pharmacotherapy. J Clin Oncol. 2016;34(35):4261-9.

5. Abudawood M. Diabetes and cancer: a comprehensive review. J Res Med Sci. 2019;24:94.

6. Siegel RL, Miller KD, Jemal A. Cancer statistics, 2020. CA Cancer J Clin. 2020;70(1):7-30

7. Popa A, Georgescu M, Popa SG, Nica AE, Georgescu EF. New insights in the molecular pathways linking obesity, type 2 diabetes and cancer. Rom J Morphol Embryol. 2019;60(4):1115-25.
8 . de Kort S, Simons $C$ van den Brandt PA, Janssen-Heijnen MLG, Sanduleanu S, Masclee AAM, et al. Diabetes mellitus, genetic variants in the insulin-like growth factor pathway and colorectal cancer risk. Int J Cancer. 2019;145(7):1774-81.

9. Zhu T, Zhu Y, Xuan Y, Gao H, Cai X, Piersma SR, et al. DPHL: a DIA panhuman protein mass spectrometry library for robust biomarker discovery. Genomics Proteomics Bioinformatics. 2020;18(2):104-19.

10. Xu M, Deng J, Xu K, Zhu T, Han L, Yan Y, et al. In-depth serum proteomics reveals biomarkers of psoriasis severity and response to traditional Chinese medicine. Theranostics. 2019;9(9):2475-88.

11. Geyer PE, Kulak NA, Pichler G, Holdt LM, Teupser D, Mann M. Plasma Proteome Profiling to Assess Human Health and Disease. Cell Syst. 2016;2(3):185-95.

12. Gillet LC, Navarro P, Tate $S$, Rost $H$, Selevsek N, Reiter $L$, et al. Targeted data extraction of the MS/MS spectra generated by data-independent acquisition: a new concept for consistent and accurate proteome analysis. Mol Cell Proteomics. 2012;11(6):0111 016717

13. Sahni S, Krisp C, Molloy MP, Nahm C, Maloney S, Gillson J, et al. PSMD11, PTPRM and PTPRB as novel biomarkers of pancreatic cancer progression. Biochim Biophys Acta Gen Subj. 2020;1864(11):129682.

14. Bouchal P, Schubert OT, Faktor J, Capkova L, Imrichova H, Zoufalova K, et al. Breast cancer classification based on proteotypes obtained by SWATH mass spectrometry. Cell Rep. 2019;28(3):832-43 e7.

15. Min L, Zhu S, Wei R, Zhao Y, Liu S, Li P, et al. Integrating SWATH-MS proteomics and transcriptome analysis identifies CHI3L1 as a plasma biomarker for early gastric cancer. Mol Ther Oncolytics. 2020;17:257-66.

16. Li T, Fan J, Wang B, Traugh N, Chen Q, Liu JS, et al. TIMER: a web server for comprehensive analysis of tumor-infiltrating immune cells. Cancer Res. 2017:77(21):e108-10.

17. Uhlen M, Oksvold P, Fagerberg L, Lundberg E, Jonasson K, Forsberg M, et al. Towards a knowledge-based human protein atlas. Nat Biotechnol. 2010;28(12):1248-50.

18. Uhlen M, Fagerberg L, Hallstrom BM, Lindskog C, Oksvold P, Mardinoglu A, et al. Proteomics tissue-based map of the human proteome. Science. 2015:347(6220):1260419.

19. Huang $H$, Dong $X$, Kang $M X, X u B$, Chen $Y$, Zhang B, et al. Novel blood biomarkers of pancreatic cancer-associated diabetes mellitus identified by peripheral blood-based gene expression profiles. Am J Gastroenterol. 2010;105(7):1661-9.

20. Lee JY, Jeon I, Lee JM, Yoon JM, Park SM. Diabetes mellitus as an independent risk factor for lung cancer: a meta-analysis of observational studies. Eur J Cancer. 2013:49(10):2411-23.

21. Tseng $\mathrm{CH}$. Higher risk of mortality from lung cancer in Taiwanese people with diabetes. Diabetes Res Clin Pract. 2013;102(3):193-201.

22. Huang L, Wang L, Hu X, Chen S, Tao Y, Su H, et al. Machine learning of serum metabolic patterns encodes early-stage lung adenocarcinoma. Nat Commun. 2020:11(1):3556.

23. Zhang J, Gold KA, Lin HY, Swisher SG, Xing Y, Lee JJ, et al. Relationship between tumor size and survival in non-small-cell lung cancer (NSCLC) an analysis of the surveillance, epidemiology, and end results (SEER) registry. J Thorac Oncol. 2015;10(4):682-90.

24. Keegan A, Ricciuti B, Garden P, Cohen L, Nishihara R, Adeni A, et al. Plasma IL-6 changes correlate to PD-1 inhibitor responses in NSCLC. J Immunother Cancer. 2020;8(2):e000678.

25. Potprommanee L, Ma HT, Shank L, Juan YH, Liao WY, Chen ST, et al. GM2-activator protein: a new biomarker for lung cancer. J Thorac Oncol. 2015:10(1):102-9.

26. Chen Z, Wang Y, Fang M. Analysis of tumor markers in pleural effusion and serum to verify the correlations between serum tumor markers and tumor size, TNM stage of lung adenocarcinoma. Cancer Med. 2020;9(4):1392-9.

27. Zhang B, Whiteaker JR, Hoofnagle AN, Baird GS, Rodland KD, Paulovich AG. Clinical potential of mass spectrometry-based proteogenomics. Nat Rev Clin Oncol. 2019:16(4):256-68.

28. Indovina P, Marcelli E, Pentimalli F, Tanganelli P, Tarro G, Giordano A. Mass spectrometry-based proteomics: the road to lung cancer biomarker discovery. Mass Spectrom Rev. 2013;32(2):129-42.

29. Zheng X, Xu K, Zhou B, Chen T, Huang Y, Li Q, et al. A circulating extracellular vesicles-based novel screening tool for colorectal cancer revealed by shotgun and data-independent acquisition mass spectrometry. J Extracell Vesicles. 2020;9(1):1750202. 
30. Martinez-Garcia E, Lesur A, Devis L, Cabrera S, Matias-Guiu X, Hirschfeld $M$, et al. Targeted proteomics identifies proteomic signatures in liquid biopsies of the endometrium to diagnose endometrial cancer and assist in the prediction of the optimal surgical treatment. Clin Cancer Res. 2017;23(21):6458-67.

31. Rifai N, Gillette MA, Carr SA. Protein biomarker discovery and validation: the long and uncertain path to clinical utility. Nat Biotechnol. 2006;24(8):971-83.

32. Kashiwagi $H$, Ishimoto H, Izumi SI, Seki T, Kinami R, Otomo A, et al. Human PZP and common marmoset A2ML1 as pregnancy related proteins. Sci Rep. 2020;10(1):5088.

33. Helgason S, Damber MG, von Schoultz B, Stigbrand T. Estrogenic potency of oral replacement therapy estimated by the induction of pregnancy zone protein. Acta Obstet Gynecol Scand. 1982;61(1):75-9.

34. Finch S, Shoemark A, Dicker AJ, Keir HR, Smith A, Ong S, et al. Pregnancy zone protein is associated with airway infection, neutrophil extracellular trap formation, and disease severity in bronchiectasis. Am J Respir Crit Care Med. 2019;200(8):992-1001.

35. Su L, Zhang G, Kong X. Prognostic significance of pregnancy zone protein and its correlation with immune infiltrates in hepatocellular carcinoma. Cancer Manag Res. 2020;9(12):9883-91.

36. Theodoraki MN, Yerneni S, Gooding WE, Ohr J, Clump DA, Bauman JE, et al. Circulating exosomes measure responses to therapy in head and neck cancer patients treated with cetuximab, ipilimumab, and IMRT. Oncoimmunology. 2019;8(7):1593805.

\section{Publisher's Note}

Springer Nature remains neutral with regard to jurisdictional claims in published maps and institutional affiliations.
Ready to submit your research? Choose BMC and benefit from:

- fast, convenient online submission

- thorough peer review by experienced researchers in your field

- rapid publication on acceptance

- support for research data, including large and complex data types

- gold Open Access which fosters wider collaboration and increased citations

- maximum visibility for your research: over $100 \mathrm{M}$ website views per year

At BMC, research is always in progress.

Learn more biomedcentral.com/submissions 